\title{
Deep brain stimulation of the cerebellum for poststroke motor rehabilitation: from laboratory to clinical trial
}

\author{
Connor A. Wathen, MD, ${ }^{1}$ Leonardo A. Frizon, MD, ${ }^{2}$ Tanmoy K. Maiti, MD, ${ }^{3}$ Kenneth B. Baker, PhD, ${ }^{4}$ \\ and Andre G. Machado, MD, PhD ${ }^{3}$ \\ ${ }^{1}$ Cleveland Clinic Lerner College of Medicine; ${ }^{2}$ Center for Neurological Restoration, Neurological Institute, Cleveland Clinic; \\ ${ }^{3}$ Department of Neurosurgery, Neurological Institute, Cleveland Clinic; and ${ }^{4}$ Department of Neurosciences, Lerner Research \\ Institute, Cleveland Clinic, Cleveland, Ohio
}

\begin{abstract}
Ischemic stroke is a leading cause of disability worldwide, with profound economic costs. Poststroke motor impairment is the most commonly encountered deficit resulting in significant disability and is the primary driver of stroke-associated healthcare expenditures. Although many patients derive some degree of benefit from physical rehabilitation, a significant proportion continue to suffer from persistent motor impairment. Noninvasive brain stimulation, vagal nerve stimulation, epidural cortical stimulation, and deep brain stimulation (DBS) have all been studied as potential modalities to improve upon the benefits derived from physical therapy alone. These neuromodulatory therapies aim primarily to augment neuroplasticity and drive functional reorganization of the surviving perilesional cortex.

The authors have proposed a novel and emerging therapeutic approach based on cerebellar DBS targeted at the dentate nucleus. Their rationale is based on the extensive reciprocal connectivity between the dentate nucleus and wide swaths of cerebral cortex via the dentatothalamocortical and corticopontocerebellar tracts, as well as the known limitations to motor rehabilitation imposed by crossed cerebellar diaschisis. Preclinical studies in rodent models of ischemic stroke have shown that cerebellar DBS promotes functional recovery in a frequency-dependent manner, with the most substantial benefits of the therapy noted at $30-\mathrm{Hz}$ stimulation. The improvements in motor function are paralleled by increased expression of markers of synaptic plasticity, synaptogenesis, and neurogenesis in the perilesional cortex. Given the findings of preclinical studies, a first-in-human trial, Electrical Stimulation of the Dentate Nucleus Area (EDEN) for Improvement of Upper Extremity Hemiparesis Due to Ischemic Stroke: A Safety and Feasibility Study, commenced in 2016. Although the existing preclinical evidence is promising, the results of this Phase I trial and subsequent clinical trials will be necessary to determine the future applicability of this therapy.
\end{abstract}

https://thejns.org/doi/abs/10.3171/2018.5.FOCUS18164

KEYWORDS stroke; deep brain stimulation; cerebellum; rehabilitation

$\mathrm{I}$ SCHEMIC stroke is a leading cause of death and disability worldwide. Approximately 795,000 new or recurrent strokes are reported annually in the United States, with $87 \%$ of strokes resulting from cerebral ischemia. ${ }^{46}$ Over the past decade, death from stroke has declined across all age groups. More rapid diagnosis and treatment with newer therapies such as intravenous tissue plasminogen activator and thrombectomy will hopefully ensure that this trend continues. As stroke-related mortality continues to decline, however, the number of individuals with post- stroke disability may increase. Of the many neurological impairments that may develop following ischemic stroke, upper-extremity weakness is the most debilitating, impairing patients' ability to care for themselves and leaving them reliant on caregivers. ${ }^{62}$

Following an ischemic stroke, most patients will spontaneously regain some degree of lost motor function. Early implementation of physical therapy has been repeatedly shown to further improve motor recovery. ${ }^{14,15,59}$ Unfortunately, the window of opportunity during which such gains

ABBREVIATIONS CCD = crossed cerebellar diaschisis; $\mathrm{CPC}=$ cerebropontocerebellar; $\mathrm{DBS}=$ deep brain stimulation; $\mathrm{DN}=$ dentate nucleus; $\mathrm{DTC}=$ dentatothalamocortical; ECS = epidural cortical stimulation; EDEN = Electrical Stimulation of the Dentate Nucleus Area; LCN = lateral cerebellar nucleus; LTP = long-term potentiation; MEP = motor evoked potential; NIBS = noninvasive brain stimulation; NMDA = N-methyl-D-aspartate; VNS = vagal nerve stimulation.

SUBMITTED March 31, 2018. ACCEPTED May 21, 2018.

INCLUDE WHEN CITING DOI: 10.3171/2018.5.FOCUS18164. 
can be realized is typically limited to the 3-6 months following the stroke, after which residual deficits typically stabilize. ${ }^{5,63}$ As a result, even with the benefits provided by physical rehabilitation, many patients remain significantly impaired and incapable of caring for themselves without assistance. In addition to reducing functional independence, poststroke motor impairments are also associated with increased healthcare expenditures. In the United States, the indirect costs associated with stroke are expected to rise from $\$ 25$ billion in 2010 to $\$ 44$ billion in $2030 .{ }^{25}$ Direct expenses related to stroke are anticipated to increase more than 3-fold-from \$71.6 to \$184.1 billion-during the same time period. To reduce the burden of physical disability and alleviate the enormous burden poststroke care places on the healthcare system, more effective therapies are needed to reduce chronic poststroke motor deficits.

Deep brain stimulation (DBS) is a potential therapeutic modality to aid in the restoration of motor function after stroke. Currently, DBS is approved by the FDA for the treatment of essential tremor and Parkinson disease. Humanitarian device exemptions granted by the FDA have enabled the use of DBS in the treatment of dystonia and obsessive-compulsive disorder as well. Furthermore, ongoing investigations into the use of DBS in the treatment of Alzheimer disease, ${ }^{40}$ traumatic brain injury, ${ }^{23}$ obesity ${ }^{65}$ depression, ${ }^{16}$ and anorexia nervosa ${ }^{39}$ among others, seek to expand the indications for this therapy. ${ }^{45}$ The utility of DBS stems from the ability to modulate a wide variety of structures, depending on the targeted region for stimulation. With respect to stroke, our group has investigated over the span of 10 years the use of DBS of the dentatothalamocortical (DTC) pathway to augment poststroke motor rehabilitation. ${ }^{41,64}$ The ongoing translational and clinical research supporting the use of neuromodulationbased approaches in stroke will be reviewed here, with a specific emphasis on dentate nucleus (DN) DBS-based treatment.

\section{Neuroplasticity After Stroke}

Neuroplasticity is believed to be the primary mechanism by which poststroke motor recovery occurs, with changes at the synaptic, cellular, and network levels contributing to reorganization of the surviving neural tissue and mediating recovery. Changes in the perilesional cortex as well as in homotopic regions of the contralesional hemisphere have been shown to help facilitate motor recovery. ${ }^{26,29}$ At the network level, functional recovery is associated with reorganization of the somatotopy of the primary motor cortex ${ }^{32,37,49}$ and increases in cortical excitability. ${ }^{37}$ Reorganization of perilesional cortex and functional motor improvements are thus thought to be mediated in part by increased neuronal excitability leading to neuroplastic changes. Underscoring the importance of this process, motor representation in the perilesional cortex and the area devoted to forelimb function have been correlated with recovery of function after stroke. ${ }^{22}$

Long-term potentiation (LTP) is a key driver of the synaptic changes that facilitate poststroke recovery. ${ }^{19}$ The central concept underlying LTP is Hebbian plasticity, which occurs when repetitive activation by one neuron drives metabolic and morphological adaptations in the target neuron that increase the efficiency through which the first neuron activates the second. ${ }^{24}$ The postsynaptic $N$-methylD-aspartate (NMDA) glutamate receptor, CaMKII, and PKC have been shown to be important mediators of LTP. ${ }^{9}$ Changes are not restricted to existing synapses, however; there is evidence to suggest that recovery is associated with the formation of new synapses, as evidenced by animal models demonstrating increased synaptophysin expression in the perilesional cortex. ${ }^{44}$ Synaptogenesis and modulation of synaptic strength through LTP are essential in motor learning and motor recovery following stroke.

Axonal sprouting and, to a lesser extent, neurogenesis may also contribute to functional reorganization and recovery after stroke. ${ }^{9}$ Axonal sprouting occurs both in the perilesional area and in regions distant from the infarct. ${ }^{8}$ Axonal sprouting is guided by multiple factors, which act to direct and support growth of the axon. Axonal growth is inhibited by NogoA, EphrinA5, and chondroitin sulfate proteoglycans, whereas GDF10, GAP43, and numerous other cell adhesion, axonal guidance, and cytoskeletal molecules enable growth and sprouting. ${ }^{36}$ Supplementing these processes, neuronal stem cells, guided by chemokines and growth factors from newly formed vessels, migrate from the subventricular zone to the core of the infarct. ${ }^{21}$ Unfortunately, the magnitude of this neurogenic response is quite small, and there is little evidence to suggest that this response contributes significantly to functional recovery in humans.

\section{Non-DBS-Based Neuromodulation Approaches to Enhance Poststroke Motor Recovery}

Several neuromodulatory approaches to poststroke motor recovery have been studied extensively. The lessons learned from these studies have greatly informed the development of DBS of the DTC pathway. ${ }^{55}$ Transcranial magnetic stimulation of the motor cortex, one of the most widely used noninvasive brain stimulation (NIBS) modalities, has been used as a prognostic tool to determine integrity of descending motor pathways ${ }^{47,54}$ and to map changes in motor cortex representation during rehabilitation. ${ }^{6}$ Repetitive transcranial magnetic stimulation is currently under extensive investigation to examine its effects on motor reorganization and recovery after stroke. ${ }^{27} \mathrm{Al}-$ though widespread adoption of NIBS remains hampered by modest therapeutic effect sizes to date and limited patient access to the technology, use of NIBS has aided in the development of a strong evidence base in support of more invasive therapies..$^{53}$

Implantable neurostimulation devices circumvent several of the shortcomings of NIBS by allowing direct, long-term stimulation of the nervous system. Preclinical studies of vagal nerve stimulation (VNS) have shown that stimulation produces numerous physiological changes, including changes in neurotrophic signaling, neuroinflammation, and excitotoxicity in addition to modulation of cerebral blood flow. ${ }^{7}$ In rodent models of ischemic stroke, VNS paired with physical rehabilitation led to significant 
A

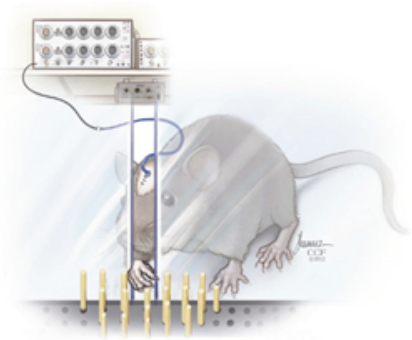

B

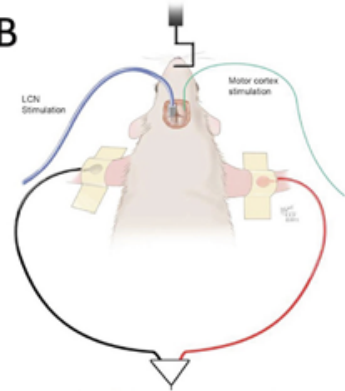

C

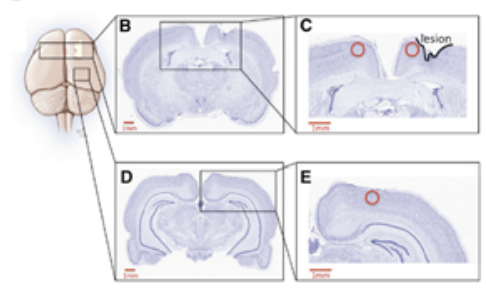

FIG. 1. Timeline of preclinical studies investigating LCN DBS for poststroke motor rehabilitation. A: Illustration shows an animal executing the pasta matrix task used to evaluate motor function and recovery. (Originally published in Machado et al., $2013,{ }^{43}$ and then in Cooperrider et al., 2014. ${ }^{11}$ ) B: Illustration shows the experimental setup used to enable concurrent LCN DBS, intracortical microstimulation, and recording of MEPs. (Originally published in Baker et al., 2010, ${ }^{3}$ and then in Park et al., 2015. ${ }^{50}$ ) C: Illustration shows the sites at which expression of markers of neuroplasticity were quantified. (Originally published in Cooperrider et al., 2014.11) Reprinted with permission, Cleveland Clinic Center for Medical Art \& Photography (c 2012-2018. All rights reserved.

improvements in motor function relative to controls receiving rehabilitation alone. ${ }^{30}$ These preclinical findings formed the basis for a recent clinical trial in the United Kingdom. ${ }^{13}$ In this trial, 9 patients were randomized to VNS plus rehabilitation and 11 were assigned to rehabilitation alone. Although the intention-to-treat analysis did not reveal any significant difference in upper-extremity function as measured via the Fugl-Meyer Assessment upper extremity score, the per-protocol analysis showed some improvements in the VNS-treated group relative to the rehabilitation alone control group (between-group difference, 6.5; 95\% CI 0.4-12.6). Additional studies are necessary to clarify the utility of VNS in the treatment of poststroke motor deficits.

Early studies of epidural cortical stimulation (ECS) for poststroke motor rehabilitation were promising, with both rodent and nonhuman primate studies showing significant motor improvements following ECS. ${ }^{1,33,51,60}$ Based on preclinical evidence and the results of Phase I and II clinical trials, ${ }^{4,34}$ a pivotal Phase III trial named Safety and Effectiveness of Cortical Stimulation in the Treatment of Stroke Patients With Upper Extremity Hemiparesis (EVEREST) was conducted in which 146 patients participated (91 treatment, 55 control). ${ }^{35}$ Unfortunately, no difference was seen in motor recovery between the ECS plus rehabilitation group and the rehabilitation alone group. ${ }^{35,52}$ In a subsequent analysis, patients who responded favorably to ECS were found to have a significantly greater proportion of intact corticospinal tract and were more likely to demonstrate preserved motor-evoked responses to cortical stimulation than nonresponders. ${ }^{48}$ Subsequent studies have demonstrated the importance of infarct location, the timing of stimulation relative to the ischemic insult, the preservation of descending motor pathways, and the need to stimulate wide areas of perilesional cortex to produce benefits in motor function.

\section{Deep Brain Stimulation for Stroke Recovery}

Elias et al. recently published a comprehensive review of the literature that aimed to assess the potential use of DBS for recovery from poststroke conditions. The primary aim of the majority of the studies that they identified was relief of other stroke sequelae, including pain, dystonia, and spasticity. With regard to poststroke hemiparesis, only 9 studies, including a total of only 20 patients, have reported the effects of DBS on motor recovery, and none of them included motor function as a primary outcome. Overall, the authors found that the posterior limb of the internal capsule was the target most associated with improvement in paresis in poststroke patients. Numerous problems arise in interpreting these data, however-most notably the fact that these studies were designed to treat poststroke pain, not motor deficits. ${ }^{20}$

We were the first group to propose and investigate the use of DBS of the DTC pathway for poststroke rehabilitation in preclinical models and, more recently, in a first-inhuman clinical trial. Our approach carries the potential to address many of the pitfalls and shortcomings of previously investigated neuromodulation therapies for stroke rehabilitation. Its primary strength lies in its ability to modulate wide swaths of perilesional cortex by targeting a compact nodal point in a network with dense projections to a variety of cerebral subcortical and cortical regions. The theoretical underpinnings, preclinical evidence, and ongoing clinical studies regarding cerebellar DBS are reviewed here (Fig. 1).

\section{Anatomical and Pathophysiological Rationale}

The robust reciprocal connections between the cerebral cortex and the contralateral cerebellar hemisphere through the cerebropontocerebellar (CPC) and DTC fibers demonstrate the strong interdependence of these structures (Fig. 2). Following a large cortical insult, such as an ischemic stroke, decreased afferent input to the cerebellum secondary to disruption of the CPC tract leads to decreased cerebellar metabolism, blood flow, and activity. This deafferentation and reduced activity of the cerebellum then results in decreased excitatory output from the cerebellum to the affected cortex via the reciprocal DTC pathway. This phenomenon, called crossed cerebellar diaschisis (CCD), is a critical component of the rationale underlying the selection of the DTC tract at the DN as a target to enhance poststroke motor recovery. ${ }^{28}$ The incidence of CCD following stroke is variable, with reports 

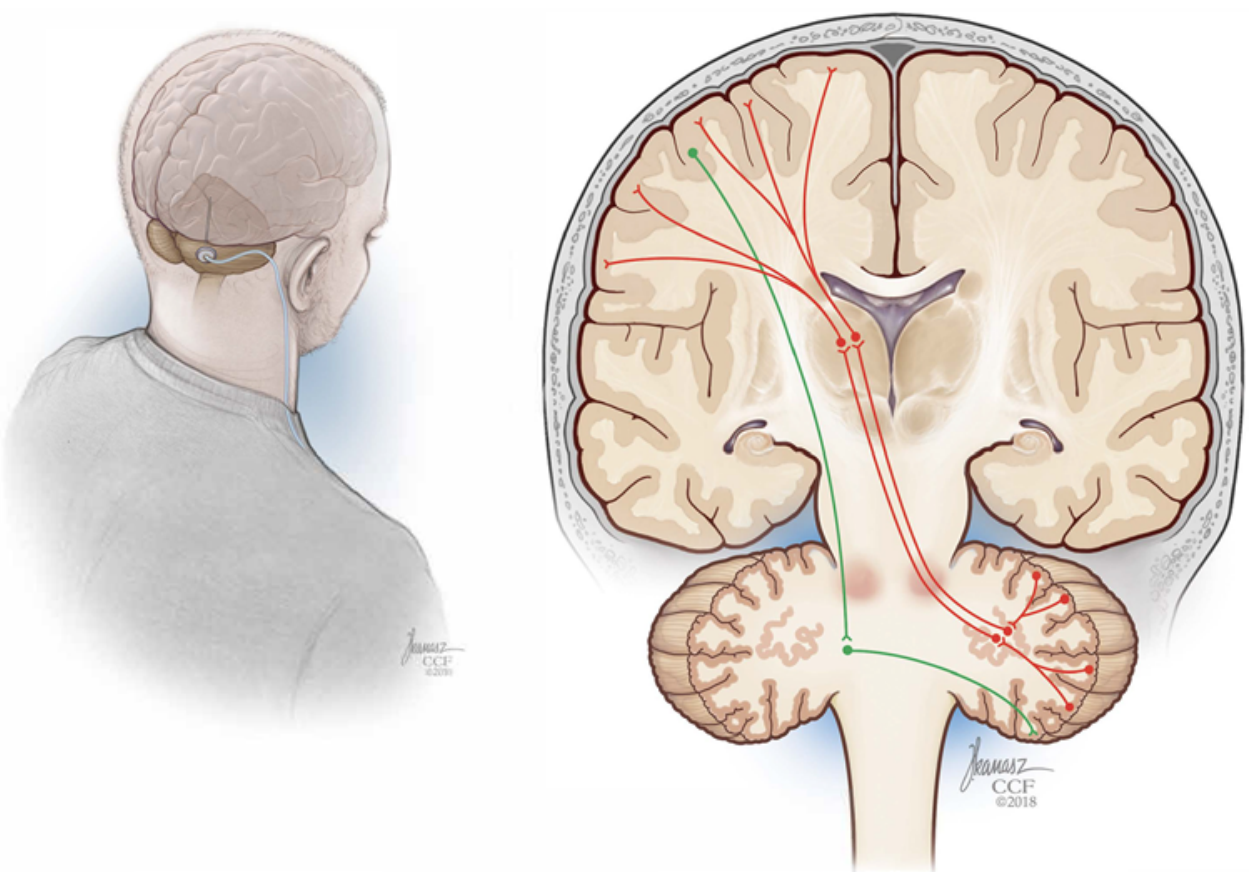

FIG. 2. Anatomy of human DTC and CPC tracts. The DTC pathway is shown in red projecting through the ipsilateral superior cerebellar peduncle, decussating at the pons, and then terminating in the contralateral thalamus before projecting to a wide array of cortical targets. The CPC pathway is shown in green descending from the cortex, decussating in the ipsilateral pons, and terminating in the contralateral DN. Reprinted with permission, Cleveland Clinic Center for Medical Art \& Photography @ 2012-2018. All rights reserved.

ranging from $16 \%$ to greater than $50 \%$ in PET and SPECT imaging studies. ${ }^{38,58,66}$ Evidence suggests that CCD is associated with increased stroke severity, and the finding of CCD in the early subacute phase following stroke correlates with worse motor outcomes and decreased cortical excitability. $28,31,56,58$

The DN, situated adjacent to the fourth ventricle, is the largest of the deep cerebellar nuclei (Fig. 3). ${ }^{2}$ The nucleus is divided into a dorsal motor domain that projects primarily to motor and premotor cortex, and a ventral nonmotor domain that projects to prefrontal and parietal cortex. ${ }^{17}$ The DN receives input from the lateral cerebellar hemisphere as well as the CPC tract by way of various pontine nuclei. Its primary output is the DTC tract, which exits the cerebellum via the ipsilateral superior cerebellar peduncle and decussates in the midbrain before synapsing in the contralateral thalamus via several nuclei, including the ventral lateral pars caudalis, ventral posterior lateral pars oralis, and medial dorsal. ${ }^{61}$ From the thalamus, the DTC tract then projects to several cortical regions, including the premotor, primary motor, and parietal cortices ${ }^{17,18}$ Given the functional relevance of the reciprocal connectivity between the cerebellum and cerebral cortex illustrated by $\mathrm{CCD}$, and the widespread disynaptic excitatory projections of the DN to the cerebral cortex, cerebellar DBS holds the potential to influence large perilesional cortical areas, with the hypothesized effect of promoting functional reorganization and, in turn, motor recovery.

\section{Preclinical Evidence}

The first proof-of-principle study investigating this hypothesis was published by our group in $2009 .{ }^{42}$ In this study, rats were pretrained on the Montoya staircase task before stroke induction, which was conducted using the 3 -vessel occlusion approach. Animals were then treated with sham, $10-\mathrm{Hz}, 20-\mathrm{Hz}$, or $50-\mathrm{Hz}$ stimulation of the contralesional lateral cerebellar nucleus (LCN), the rodent analog to the human DN. Relative to the sham-stimulated animals, those receiving chronic $20-\mathrm{Hz}$ stimulation demonstrated significant improvements in motor function as indexed by performance on the Montoya staircase task.

Given the frequency-dependent nature of the improvements seen in the first study and the understanding that increased cortical excitability underlies neuroplastic changes in the cortex, a subsequent study was performed in which concurrent LCN DBS and intracortical microstimulation of the contralateral primary motor cortex were performed in naïve rats. ${ }^{3} \mathrm{LCN}$ DBS was again delivered over a range of frequencies, including $20 \mathrm{~Hz}, 30 \mathrm{~Hz}, 40 \mathrm{~Hz}, 50 \mathrm{~Hz}$, or $100 \mathrm{~Hz}$, and the amplitude of the intracortical microstimulation-induced motor evoked potential (MEP) measured across the hindlimb was used to index cortical excitability. The results showed that $30-\mathrm{Hz}$ stimulation resulted in the most sustained facilitation of MEPs, whereas $100-\mathrm{Hz}$ stimulation actually reduced excitability over time. A later study confirmed that although MEPs were less robust in poststroke animals relative to naïve controls, LCN DBS consistently produced frequency-specific modulation of cortical excitability. ${ }^{50}$

After demonstrating that LCN DBS can promote motor recovery and determining optimal stimulation parameters, studies into the mechanistic underpinnings of the therapy 

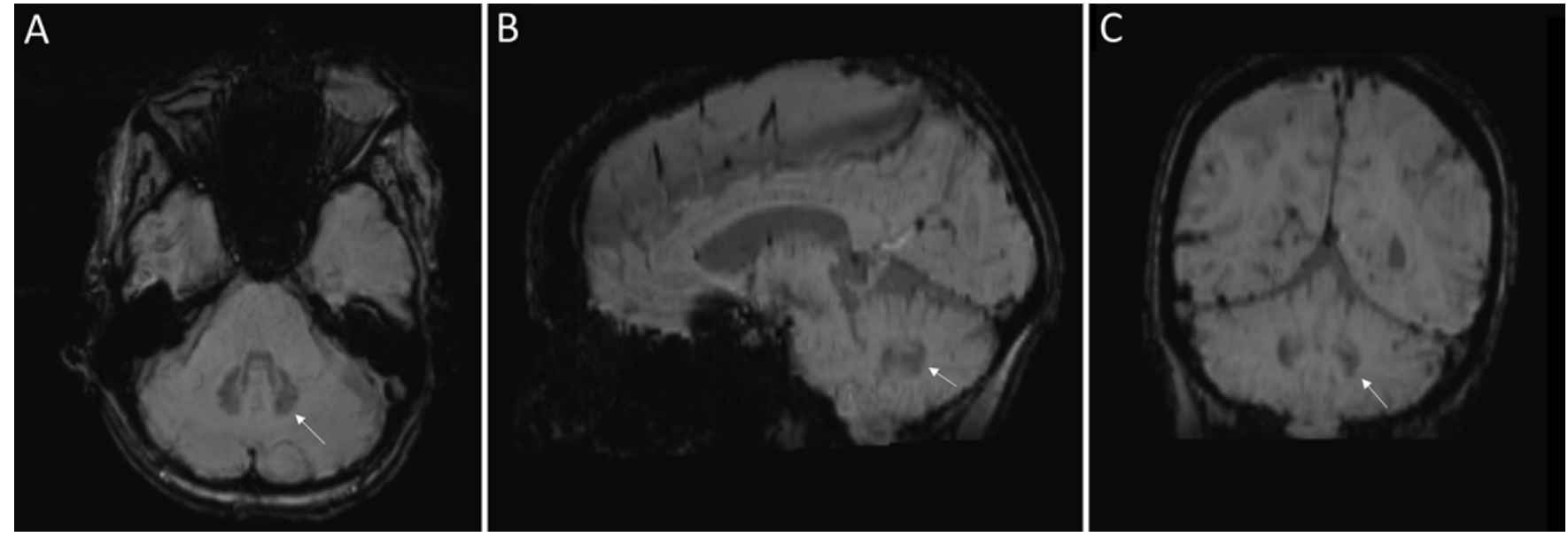

FIG. 3. 3T MRI of the DN. Axial (A), sagittal (B), and coronal (C) views of the DN from a 3-T susceptibility-weighted image (SWI). The DN is indicated by the white arrows.

were undertaken. Immunohistochemical analysis of the perilesional cortex found that the motor benefits of LCN DBS, measured via the pasta matrix task (Fig. 1), were accompanied by increased expression of synaptophysin, a marker of synaptogenesis. ${ }^{43}$ Building on this finding, we investigated the effects of chronic $30-\mathrm{Hz}$ stimulation, a burst stimulation pattern, and sham stimulation on motor recovery, perilesional expression of markers of LTP, and reorganization of the motor cortex..$^{11}$ In line with previous studies, the efficacy of LCN DBS on motor recovery was once again shown on the pasta matrix task. Western blot analysis of perilesional tissue also demonstrated increased expression of several markers of LTP in stimulated animals-specifically synaptophysin, the NMDA receptor, PSD95, and CamKII. Paralleling this increased expression of markers of neuroplasticity, a 2-fold increase in synaptic density in the perilesional cortex of treated animals was seen via 3D electron microscopy. Finally, intracortical microstimulation of the primary motor cortex showed that stroke induced a significant reduction in the amount of cortex devoted to the forelimb relative to naïve controls. Treatment with LCN DBS resulted in significant expansion of the poststroke forelimb representation relative to sham-stimulated animals. In addition to inducing synaptogenesis and LTP, LCN DBS may also promote neurogenesis in rodent models of stroke. Chan et al. showed that following stroke, LCN DBS-treated animals showed an increased number of cells coexpressing BRDU, a marker of cell proliferation, and several neurogenic markers, indicating the presence of neurogenesis. ${ }^{10}$ Interestingly, the neurogenic effect of LCN DBS appeared to be selective for excitatory, glutamatergic neurons relative to inhibitory GABAergic neurons. Combined, the findings of these studies have provided evidence in support of the ability of LCN DBS to promote motor recovery, neuroplasticity, and functional reorganization of the motor cortex.

\section{Ongoing Research and Future Directions} Clinical Studies

Given the preclinical evidence in support of DBS of the
DTC pathway, an investigational device exemption (IDE \# G150237) was granted to conduct a Phase I trial of DBS of the DN area. Supported by funding from the BRAIN Initiative, a first-in-human trial, Electrical Stimulation of the Dentate Nucleus Area (EDEN) for Improvement of Upper Extremity Hemiparesis Due to Ischemic Stroke: A Safety and Feasibility Study (NCT02835443; see ClinicalTrials. gov), began enrolling patients in 2016. The EDEN study is enrolling patients between the ages of 25 and 75 years who have experienced a single ischemic stroke within the previous 12-36 months and are still experiencing moderate to severe unilateral upper-extremity paresis. In addition to the primary intervention of physical rehabilitation combined with DN stimulation, patients undergo a battery of tests with the goal of collecting data to hone future studies. This includes structural MR imaging, metabolic imaging to detect changes in the cerebellum and cerebral cortex, local field potential recordings from the DN during a lead externalization period, characterization of cortical excitability over time, and changes in perilesional cortical maps. Ancillary data from this study will aid in refining patient selection, DBS targeting, and optimal stimulation parameters to facilitate recovery and minimize side effects. Pending the results of the ongoing Phase I trial, future randomized controlled trials will be necessary to evaluate the ultimate utility of DTC DBS in promoting poststroke motor recovery and reducing disability.

\section{Preclinical Studies}

Continued preclinical investigations into LCN DBS are underway. One area of interest is the development of a closed-loop stimulation paradigm. Given the likelihood that the effects of stimulation on neural networks depend at least in part on brain state relative to motor or nonmotor tasks, we anticipate that a closed-loop system designed to deliver stimulation at critical periods of motor activity, or preceding or following motor activity, may provide greater benefit in motor recovery or minimize adverse effects of stimulation relative to continuous chronic stimulation. To this end, our group has recently shown increased beta- 
band activity in the LCN prior to the initiation of unsuccessful reaching motions compared to successful attempts, a finding that may allow for the design and development of local field potential-informed closed-loop stimulation triggering. ${ }^{12}$ Ongoing work in our laboratory is aimed at developing such a closed-loop system based on real-time detection of animal behavior.

Given the promising findings of DBS in postischemia rehabilitation, we are now extending our research to traumatic brain injury. We aim to evaluate if DBS can promote recovery of motor function after injury as well as other domains of neurological function such as learning and cognition. This work has also enabled the study of the effects of LCN DBS on postinjury apoptosis and neuroinflammation, which may provide additional mechanistic data relevant to the use of cerebellar DBS in disease models.

Optogenetic approaches to target neural circuits involved in stroke recovery are also advancing and can help inform some of the mechanisms underlying the effects of stimulation. Selective optogenetic stimulation of the contralesional LCN neurons at $10 \mathrm{~Hz}$ in Thy1-ChR2-YFP transgenic mice has now reproduced the poststroke motor rehabilitative effects first demonstrated with DBS. ${ }^{57}$

\section{Conclusions}

Preclinical evidence from rodent models of stroke demonstrate that LCN DBS promotes motor recovery that is paralleled by increased expression of markers of synaptic plasticity, synaptogenesis, neurogenesis, and reorganization of the perilesional motor cortex. Continued laboratory investigations and the ongoing Phase I EDEN trial will help determine the future direction of this promising therapy.

\section{References}

1. Adkins-Muir DL, Jones TA: Cortical electrical stimulation combined with rehabilitative training: enhanced functional recovery and dendritic plasticity following focal cortical ischemia in rats. Neurol Res 25:780-788, 2003

2. Akakin A, Peris-Celda M, Kilic T, Seker A, Gutierrez-Martin A, Rhoton A Jr: The dentate nucleus and its projection system in the human cerebellum: the dentate nucleus microsurgical anatomical study. Neurosurgery 74:401-425, 2014

3. Baker KB, Schuster D, Cooperrider J, Machado AG: Deep brain stimulation of the lateral cerebellar nucleus produces frequency-specific alterations in motor evoked potentials in the rat in vivo. Exp Neurol 226:259-264, 2010

4. Brown JA, Lutsep HL, Weinand M, Cramer SC: Motor cortex stimulation for the enhancement of recovery from stroke: a prospective, multicenter safety study. Neurosurgery 62 (Suppl 2):853-862, 2008

5. Buma F, Kwakkel G, Ramsey N: Understanding upper limb recovery after stroke. Restor Neurol Neurosci 31:707-722, 2013

6. Butler AJ, Wolf SL: Putting the brain on the map: use of transcranial magnetic stimulation to assess and induce cortical plasticity of upper-extremity movement. Phys Ther 87:719-736, 2007

7. Cai PY, Bodhit A, Derequito R, Ansari S, Abukhalil F, Thenkabail S, et al: Vagus nerve stimulation in ischemic stroke: old wine in a new bottle. Front Neurol 5:107, 2014

8. Carmichael ST: Cellular and molecular mechanisms of neural repair after stroke: making waves. Ann Neurol 59:735742,2006
9. Carmichael ST: Emergent properties of neural repair: elemental biology to therapeutic concepts. Ann Neurol 79:895906, 2016

10. Chan HH, Cooperrider J, Chen Z, Gale JT, Baker KB, Wathen CA, et al: Lateral cerebellar nucleus stimulation has selective effects on glutamatergic and GABAergic perilesional neurogenesis after cortical ischemia in the rodent model. Neurosurgery [epub ahead of print], 2017

11. Cooperrider J, Furmaga H, Plow E, Park HJ, Chen Z, Kidd $\mathrm{G}$, et al: Chronic deep cerebellar stimulation promotes longterm potentiation, microstructural plasticity, and reorganization of perilesional cortical representation in a rodent model. J Neurosci 34:9040-9050, 2014

12. Cooperrider J, Gale JT, Gopalakrishnan R, Chan HH, Wathen C, Park HJ, et al: Differential frequency modulation of neural activity in the lateral cerebellar nucleus in failed and successful grasps. Exp Neurol 277:27-34, 2016

13. Dawson J, Pierce D, Dixit A, Kimberley TJ, Robertson M, Tarver B, et al: Safety, feasibility, and efficacy of vagus nerve stimulation paired with upper-limb rehabilitation after ischemic stroke. Stroke 47:143-150, 2016

14. Dobkin BH: Strategies for stroke rehabilitation. Lancet Neurol 3:528-536, 2004

15. Dobkin BH, Dorsch A: New evidence for therapies in stroke rehabilitation. Curr Atheroscler Rep 15:331, 2013

16. Dougherty DD, Rezai AR, Carpenter LL, Howland RH, Bhati MT, O'Reardon JP, et al: A randomized sham-controlled trial of deep brain stimulation of the ventral capsule/ventral striatum for chronic treatment-resistant depression. Biol Psychiatry 78:240-248, 2015

17. Dum RP, Li C, Strick PL: Motor and nonmotor domains in the monkey dentate. Ann N Y Acad Sci 978:289-301, 2002

18. Dum RP, Strick PL: An unfolded map of the cerebellar dentate nucleus and its projections to the cerebral cortex. J Neurophysiol 89:634-639, 2003

19. Edwardson MA, Lucas TH, Carey JR, Fetz EE: New modalities of brain stimulation for stroke rehabilitation. Exp Brain Res 224:335-358, 2013

20. Elias GJB, Namasivayam AA, Lozano AM: Deep brain stimulation for stroke: current uses and future directions. Brain Stimul 11:3-28, 2018

21. Ergul A, Alhusban A, Fagan SC: Angiogenesis: a harmonized target for recovery after stroke. Stroke 43:2270-2274, 2012

22. Freundlieb N, Philipp S, Drabik A, Gerloff C, Forkert ND, Hummel FC: Ipsilesional motor area size correlates with functional recovery after stroke: a 6-month follow-up longitudinal TMS motor mapping study. Restor Neurol Neurosci 33:221-231, 2015

23. Giacino J, Fins JJ, Machado A, Schiff ND: Central thalamic deep brain stimulation to promote recovery from chronic posttraumatic minimally conscious state: challenges and opportunities. Neuromodulation 15:339-349, 2012

24. Hebb DO: The Organization of Behavior: A Neuropsychological Theory. Mahwah, NJ: L Erlbaum Assoc, 2002

25. Heidenreich PA, Trogdon JG, Khavjou OA, Butler J, Dracup $\mathrm{K}$, Ezekowitz MD, et al: Forecasting the future of cardiovascular disease in the United States: a policy statement from the American Heart Association. Circulation 123:933-944, 2011

26. Hermann DM, Chopp M: Promoting brain remodelling and plasticity for stroke recovery: therapeutic promise and potential pitfalls of clinical translation. Lancet Neurol 11:369380,2012

27. Hummel F, Cohen LG: Improvement of motor function with noninvasive cortical stimulation in a patient with chronic stroke. Neurorehabil Neural Repair 19:14-19, 2005

28. Infeld B, Davis SM, Lichtenstein M, Mitchell PJ, Hopper JL: Crossed cerebellar diaschisis and brain recovery after stroke. Stroke 26:90-95, 1995 
29. Johansen-Berg H, Rushworth MFS, Bogdanovic MD, Kischka U, Wimalaratna S, Matthews PM: The role of ipsilateral premotor cortex in hand movement after stroke. Proc Natl Acad Sci U S A 99:14518-14523, 2002

30. Khodaparast N, Hays SA, Sloan AM, Hulsey DR, Ruiz A, Pantoja M, et al: Vagus nerve stimulation during rehabilitative training improves forelimb strength following ischemic stroke. Neurobiol Dis 60:80-88, 2013

31. Kim SE, Choi CW, Yoon BW, Chung JK, Roh JH, Lee MC, et al: Crossed-cerebellar diaschisis in cerebral infarction: technetium-99m-HMPAO SPECT and MRI. J Nucl Med 38:14-19, 1997

32. Kleim JA, Barbay S, Nudo RJ: Functional reorganization of the rat motor cortex following motor skill learning. J Neurophysiol 80:3321-3325, 1998

33. Kleim JA, Bruneau R, VandenBerg P, MacDonald E, Mulrooney R, Pocock D: Motor cortex stimulation enhances motor recovery and reduces peri-infarct dysfunction following ischemic insult. Neurol Res 25:789-793, 2003

34. Levy R, Ruland S, Weinand M, Lowry D, Dafer R, Bakay $\mathrm{R}$ : Cortical stimulation for the rehabilitation of patients with hemiparetic stroke: a multicenter feasibility study of safety and efficacy. J Neurosurg 108:707-714, 2008

35. Levy RM, Harvey RL, Kissela BM, Winstein CJ, Lutsep HL, Parrish TB, et al: Epidural electrical stimulation for stroke rehabilitation: results of the prospective, multicenter, randomized, single-blinded Everest Trial. Neurorehabil Neural Repair 30:107-119, 2016

36. Li S, Overman JJ, Katsman D, Kozlov SV, Donnelly CJ, Twiss JL, et al: An age-related sprouting transcriptome provides molecular control of axonal sprouting after stroke. Nat Neurosci 13:1496-1504, 2010

37. Liepert J, Bauder H, Wolfgang HR, Miltner WH, Taub E, Weiller C: Treatment-induced cortical reorganization after stroke in humans. Stroke 31:1210-1216, 2000

38. Lin DDM, Kleinman JT, Wityk RJ, Gottesman RF, Hillis $\mathrm{AE}$, Lee AW, et al: Crossed cerebellar diaschisis in acute stroke detected by dynamic susceptibility contrast MR perfusion imaging. AJNR Am J Neuroradiol 30:710-715, 2009

39. Lipsman N, Lam E, Volpini M, Sutandar K, Twose R, Giacobbe P, et al: Deep brain stimulation of the subcallosal cingulate for treatment-refractory anorexia nervosa: 1 year follow-up of an open-label trial. Lancet Psychiatry 4:285-294, 2017

40. Lozano AM, Fosdick L, Chakravarty MM, Leoutsakos JM, Munro C, Oh E, et al: A Phase II study of fornix deep brain stimulation in mild Alzheimer's disease. J Alzheimers Dis 54:777-787, 2016

41. Machado A, Baker KB: Upside down crossed cerebellar diaschisis: proposing chronic stimulation of the dentatothalamocortical pathway for post-stroke motor recovery. Front Integr Nuerosci 6:20, 2012

42. Machado AG, Baker KB, Schuster D, Butler RS, Rezai A: Chronic electrical stimulation of the contralesional lateral cerebellar nucleus enhances recovery of motor function after cerebral ischemia in rats. Brain Res 1280:107-116, 2009

43. Machado AG, Cooperrider J, Furmaga HT, Baker KB, Park HJ, Chen Z, et al: Chronic 30-Hz deep cerebellar stimulation coupled with training enhances post-ischemia motor recovery and peri-infarct synaptophysin expression in rodents. Neurosurgery 73:344-353, 2013

44. Madinier A, Bertrand N, Rodier M, Quirié A, Mossiat C, Prigent-Tessier A, et al: Ipsilateral versus contralateral spontaneous post-stroke neuroplastic changes: involvement of BDNF? Neuroscience 231:169-181, 2013

45. Miocinovic S, Somayajula S, Chitnis S, Vitek JL: History, applications, and mechanisms of deep brain stimulation. JAMA Neurol 70:163-171, 2013

46. Mozaffarian D, Benjamin EJ, Go AS, Arnett DK, Blaha MJ, Cushman M, et al: Heart Disease and Stroke Statistics-2016
Update: a report from the American Heart Association. Circulation 133:e38-e360, 2016

47. Muellbacher W, Mamoli B: Prognostic value of transcranial magnetic stimulation in acute stroke. Stroke 26:1962-1963, 1995

48. Nouri S, Cramer SC: Anatomy and physiology predict response to motor cortex stimulation after stroke. Neurology 77:1076-1083, 2011

49. Nudo RJ, Milliken GW: Reorganization of movement representations in primary motor cortex following focal ischemic infarcts in adult squirrel monkeys. J Neurophysiol 75:21442149, 1996

50. Park HJ, Furmaga H, Cooperrider J, Gale JT, Baker KB, Machado AG: Modulation of cortical motor evoked potential after stroke during electrical stimulation of the lateral cerebellar nucleus. Brain Stimul 8:1043-1048, 2015

51. Plautz EJ, Barbay S, Frost SB, Friel KM, Dancause N, Zoubina EV, et al: Post-infarct cortical plasticity and behavioral recovery using concurrent cortical stimulation and rehabilitative training: a feasibility study in primates. Neurol Res 25:801-810, 2003

52. Plow EB, Carey JR, Nudo RJ, Pascual-Leone A: Invasive cortical stimulation to promote recovery of function after stroke: a critical appraisal. Stroke 40:1926-1931, 2009

53. Potter-Baker KA, Bonnett CE, Chabra P, Roelle S, Varnerin $\mathrm{N}$, Cunningham DA, et al: Challenges in recruitment for the study of noninvasive brain stimulation in stroke: lessons from deep brain stimulation. J Stroke Cerebrovasc Dis 25:927937, 2016

54. Rapisarda G, Bastings E, de Noordhout AM, Pennisi G, Delwaide PJ: Can motor recovery in stroke patients be predicted by early transcranial magnetic stimulation? Stroke 27:21912196, 1996

55. Schulz R, Gerloff C, Hummel FC: Non-invasive brain stimulation in neurological diseases. Neuropharmacology 64:579-587, 2013

56. Seitz RJ, Höflich P, Binkofski F, Tellmann L, Herzog H, Freund HJ: Role of the premotor cortex in recovery from middle cerebral artery infarction. Arch Neurol 55:10811088, 1998

57. Shah AM, Ishizaka S, Cheng MY, Wang EH, Bautista AR, Levy S, et al: Optogenetic neuronal stimulation of the lateral cerebellar nucleus promotes persistent functional recovery after stroke. Sci Rep 7:46612, 2017

58. Takasawa M, Watanabe M, Yamamoto S, Hoshi T, Sasaki T, Hashikawa K, et al: Prognostic value of subacute crossed cerebellar diaschisis: single-photon emission CT study in patients with middle cerebral artery territory infarct. AJNR Am J Neuroradiol 23:189-193, 2002

59. Teasell RW, Murie Fernandez M, McIntyre A, Mehta S: Rethinking the continuum of stroke rehabilitation. Arch Phys Med Rehabil 95:595-596, 2014

60. Teskey GC, Flynn C, Goertzen CD, Monfils MH, Young NA: Cortical stimulation improves skilled forelimb use following a focal ischemic infarct in the rat. Neurol Res 25:794-800, 2003

61. Tracey DJ, Asanuma C, Jones EG, Porter R: Thalamic relay to motor cortex: afferent pathways from brain stem, cerebellum, and spinal cord in monkeys. J Neurophysiol 44:532554,1980

62. Veerbeek JM, Kwakkel G, van Wegen EEH, Ket JCF, Heymans MW: Early prediction of outcome of activities of daily living after stroke: a systematic review. Stroke 42:14821488,2011

63. Verheyden G, Nieuwboer A, De Wit L, Thijs V, Dobbelaere J, Devos H, et al: Time course of trunk, arm, leg, and functional recovery after ischemic stroke. Neurorehabil Neural Repair 22:173-179, 2008

64. Wathen CA, Baker KB, Machado AG: Invasive neurostimula- 
tion for poststroke motor recovery, in Neuromodulation, ed 2. Philadelphia: 2018, pp 1147-1157

65. Whiting DM, Tomycz ND, Bailes J, de Jonge L, Lecoultre V, Wilent B, et al: Lateral hypothalamic area deep brain stimulation for refractory obesity: a pilot study with preliminary data on safety, body weight, and energy metabolism. J Neurosurg 119:56-63, 2013

66. Yamauchi H, Fukuyama H, Kimura J: Hemodynamic and metabolic changes in crossed cerebellar hypoperfusion. Stroke 23:855-860, 1992

\section{Disclosures}

Drs. Machado and Baker have distribution rights related to intellectual property in Enspire DBS, Cardionomics, and ATI. Dr.

Machado is a consultant to St. Jude Medical, and he receives clin- ical or research support for the study described (includes equipment or material) from both St. Jude Medical and the NIH. He also is on the scientific advisory board for Enspire and Medtronic.

\section{Author Contributions}

Conception and design: all authors. Drafting the article: Wathen, Frizon, Maiti. Critically revising the article: all authors. Reviewed submitted version of manuscript: all authors. Approved the final version of the manuscript on behalf of all authors: Machado. Study supervision: Machado, Baker.

\section{Correspondence}

Andre G. Machado: Cleveland Clinic, Cleveland, OH. machada@ ccf.org. 